\title{
CROWD DETECTION AND VIOLENCE PREVENTION IN REMOTE
}

\section{AREAS}

\author{
Feba K Echarankudy ${ }^{1}$, Keerthana C P ${ }^{2}$, Sajna $C^{3}$, Tiya Reba Stephen ${ }^{4}$ \\ ${ }^{1}$ Student, Dept. of Electronics and Communication \\ ${ }^{2}$ Student, Dept. of Electronics and Communication \\ ${ }^{3}$ Student, Dept. of Electronics and Communication \\ ${ }^{4}$ Assistant Professor, Dept. of Electronics and Communication
}

\begin{abstract}
Crowd is a state when number of people in an area increases in such a way that uncontrolled motion behavior of different people can cause disasters. We developed a system that is used to detect the density of crowd and give information to authorized people. The crowd detection algorithm detects crowd and the embedded section performs necessary functions if crowd is detected.
\end{abstract}

Keywords- components; crowd detection algorithm, GSM

\section{INTRODUCTION}

Crowd detection is a major area of interest in image processing domain. Intelligent surveillance systems are used for the processing of the video generated by surveillance cameras. The desirable level of applicability in real-world is not achieved fully yet. The reason is that the algorithmic assumptions about the density of objects in a scene that are often violated in the real world environment. We propose a system with particular emphasis to develop certain methods that can be operated in crowded environment. Extreme crowding results in individual loss of control, and both psychological and physiological problems. The model is used to illustrate crowd characteristics and to develop guidelines for the prevention of crowd disasters.

Crowd detection is mainly required in many of the areas where pedestrians often move in groups. These scenarios are difficult to analyze because no individual pedestrians can be properly segmented out for detection, the path people take can be quite chaotic and the background is not homogenous and hence it is difficult to distinguish humans from manmade objects. It's a serious challenge to determine the crowd using the detection algorithm. Since each scenes are rich in unique motion patterns and pedestrians are not clearly visible. A particular emphasis of this program is to develop methods that can operate in crowded environments such as prisons, public parks and schools where a large number of people can be present and interact with each other.

Computer vision algorithms were used to detect crowd []. But it proved very unsuitable in real world application. Detecting and segmenting out crowds considering the fact that basic element should look like a human [] was also studied. But all these methods were difficult to implement.

Our crowd detection algorithm uses 'vision cascade object detector' for identifying faces. It is working in Viola Jones algorithm. We used the upper frontal face as a parameter for detecting. In face detection we detect the number of faces in an image. Viola Jones algorithm considers Hear features for detecting faces. The function 'vision cascade object detector' is present in version 2012b of MATLAB.

We developed a simple system that is used to detect crowd using a MATLAB program .We also developed an embedded section which is used to send a message to a given number and produce an alarm. The two sections were used together as an excellent surveillance system.

\section{BLOCK DIAGRAM}

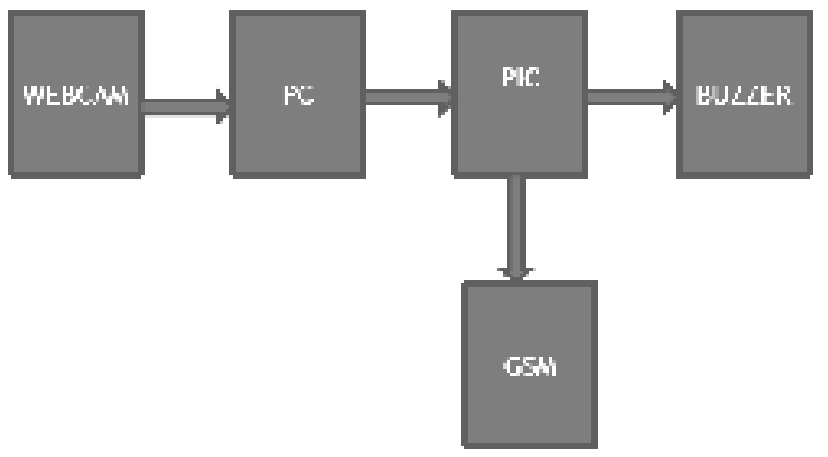

Fig 1: Block diagram showing the system

The basic block diagram of the system is shown in Figure I. It includes the embedded and the MATLAB sections. A video is taken by the webcam and the snapshot of the video is taken. A MATLAB program is running in the computer which detects crowd. If the crowd limit is exceeded a character is send to the embedded section. The embedded part has a PIC microcontroller which initiates a buzzer to crowd generate alarm and at the same time a message is send to a given number using a GSM module. This repeats for a regular interval. 


\section{CIRCUIT DIAGRAM}

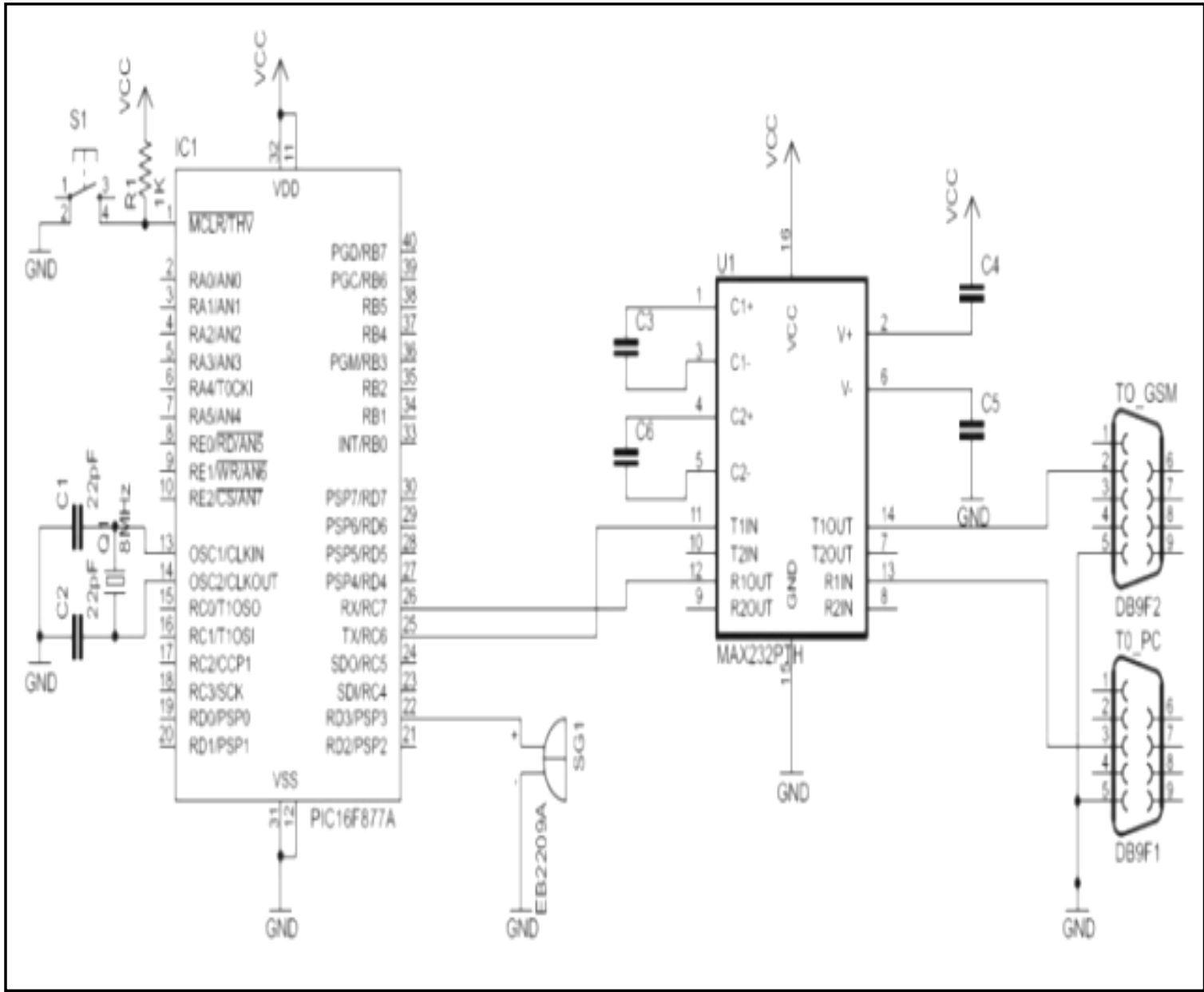

Fig 2: Circuit diagram of the system

\section{COMPONENTS DESCRIPTION}

\subsection{PIC16F877A}

The microcontroller used for the implementation of the system is PIC16F877A. The microcontroller receives the characters and it controls the entire embedded hardware.

\section{Peripheral Features}

a) Three inbuilt timers namely Timer0, Timer1, Timer2.

b) 2 Capture and control modules

c) 8 channel 10 bit ADC

d) 15 interrupt sources.

Table 1: Device Features

\begin{tabular}{|l|l|}
\hline Key Features & PIC16F877A \\
\hline EEPROM Memory & 256 bytes \\
\hline Comparators & 2 \\
\hline Interrupt sources & 15 \\
\hline ADC & 8 channel 10 bit \\
\hline Timers & 3 \\
\hline Instruction Set & 35 \\
\hline Serial Communication & USART \\
\hline
\end{tabular}

\subsection{GSM Module}

We used a SIM 900 GSM module for sending message. It supports all kinds of mobile operators.

In GSM both signaling and speech channels are digital.

The following attention commands were used in our system.

\subsubsection{AT+CMGF}

It indicates which input and output format of messages are to be used, format of messages used with send, list, read and write commands. Modes can be either PDU mode or text mode.

\subsubsection{AT+CMGS}

It is used to sends message from a TE to a network operator.

\subsection{Voltage Regulators (LM 7805)}

All circuits need a power supply. There must be a constant and smooth dc source for the efficient working of the electronic devices. A $12 \mathrm{~V}$ supply is given to the board. We use the regulating IC7805 to covert $12 \mathrm{~V}$ in to a low voltage. 
Which is shown in figure. Since the PIC works at TTL logic it is necessary to have a $5 \mathrm{~V}$ supply.LM7805 provides the necessary voltage.

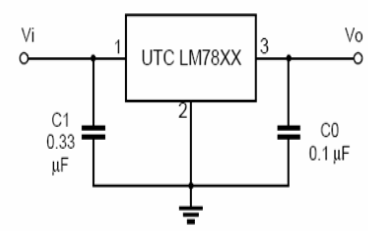

Fig 3: Voltage regulator

\subsection{MAX-232}

It is a RS232 driver IC. It is used to convert TTL logic to RS232 logic and vice versa. Typical features are listed in figure.

Table 2: RS-232 VOLTAGE LEVELS

\begin{tabular}{|l|l|l|}
\hline $\begin{array}{l}\text { RS232 Line Type \& } \\
\text { Logic Level }\end{array}$ & $\begin{array}{l}\text { RS232 } \\
\text { Voltage }\end{array}$ & $\begin{array}{l}\text { TTL } \\
\text { to/from MAX232 }\end{array}$ \\
\hline $\begin{array}{l}\text { Data Transmission } \\
(\mathrm{Rx} / \mathrm{Tx}) \text { Logic } 0\end{array}$ & $+3 \mathrm{~V}$ to & $0 \mathrm{~V}$ \\
\hline
\end{tabular}

\section{SYSTEM EXPLANATION}

We used a lucid plus camera to capture the video sequences. After 8 seconds, the snapshot of the video is taken. The image is then used to detect the number of people under the area. The built in function 'vision cascade object detector' is used to recognize the faces in the image. Also the video is then deleted since we don't want it anymore. Each face in the image is then put in a rectangular box. Figure gives the image which contains detected faces. The number of corner points of rectangle boxes is counted. The total number of corner points is divided by four which gives the number of people in the area. If this value exceeds beyond a limit then we consider it as crowd. So a character is send to the embedded section through serial port. This is repeated every 8 seconds in an infinite loop.

The PIC16F877A which is the heart of our system. It receives a signal in its receiver pin through the serial port via a $\max 232$ IC. The PIC initiates the GSM to send message to a given number saying 'CROWD DETECTED". Also the Buzzer produces alarm. Again the PIC waits for a high value at its receiver pin and the process continues.

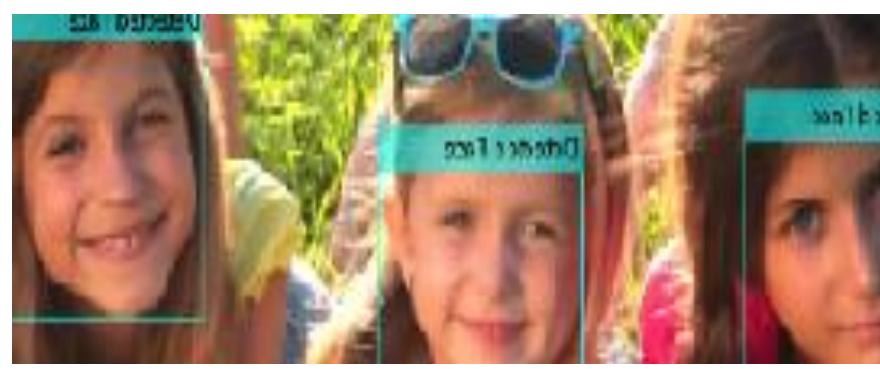

Fig 4: Output image showing detected face

\section{EXPERIMENTAL RESULTS}

Many trials were taken for different threshold values for crowd. The results were tabulated. Whenever the crowd limit was exceeded alarm was heard and message was send to the given number. Table. 3. Shows the results we obtained considering threshold as 10 .

Table 3: Results of Experiments

\begin{tabular}{|l|l|}
\hline Number of people & Alarm and message \\
\hline 10 & Yes \\
\hline 3 & No \\
\hline 8 & Yes \\
\hline
\end{tabular}

\section{FUTURE APPLICATIONS}

Though the system is used for controlling crowd, the application of the system as a security can be improved by adopting the face recognition algorithms. Also it can be used to control business done in shops by taking images from different webcams.

\section{CONCLUSION}

There are many methods for crowd detection .But the main disadvantage was the lengthy program which fails to run repeatedly. Also it takes more time. Our system uses an inbuilt function which makes it is simple and effective in detecting crowd. Also it is low cost.

\section{REFERENCES}

[1] Histograms Of Oriented Gradients For Human Detection Navneet Dalal And Bill Triggers INRIA

[2] Anil M .Cheriyadat And Richard J.Radke Detecting Dominant Motions In Dense Crowds', ,Senior Member, IEEE.

[3] Crowd Detection from Still Images Ognjen Arandjlovi'c. Department Of Engineering, University Of Cambridge ,Cb2 Ipz.

[4] Crowd Detection In Video Sequence Pini.Reisman Ofer .Mano Shai.Avidan Amnon.Shashua,2004 IEEE

[5] Density Aware Person Detection And Tracking In Crowds.Mikel Rod Riguez 1, 4 Ivan Laptev2, 4 Josef Sivic 2,4 Jean -Yves Audibert 3,41' Ecole Normale Sup Erieure 2inria 3 Imagine,Ligma,Universite ParisEst.. 